\title{
On the state-space realization of 2-periodic image behaviors
}

\author{
Diego Napp \\ CIDMA, Department of Mathematics \\ University of Aveiro \\ Aveiro, Portugal \\ diego@ua.pt
}

\author{
Paula Rocha \\ SYSTEC, Faculty of Engineering \\ University of Porto \\ Porto, Portugal \\ mprocha@fe.up.pt
}

\author{
Ricardo Pereira \\ CIDMA, Department of Mathematics \\ University of Aveiro \\ Aveiro, Portugal \\ ricardopereira@ua.pt
}

\author{
Raquel Pinto \\ CIDMA, Department of Mathematics \\ University of Aveiro \\ Aveiro, Portugal \\ raquel@ua.pt
}

\begin{abstract}
In this paper we study the realization of periodically time-varying behavioral systems by means of periodic state-space models. In particular, we focus on the case of period two and investigate under which conditions a given behavior with periodic representation obtained by alternating two time-invariant image representations can be realized by a periodic state-space system. We first show that, in general, one cannot expect to obtain a periodic state-space realization by means of the individual realizations of each associated time-invariant behaviors. However, we give conditions for such procedure to hold. The presented results are illustrated by examples.
\end{abstract}

Index Terms-Behavioral systems, Periodically time-varying systems, State-space representations.

\section{INTRODUCTION}

Traditionally, mathematicians and engineers are used to develop modelling and control of physical systems within the framework of input/output thinking. Such a framework is, of course, often perfectly suitable to describe the interaction between a system and its environment. It is indeed appealing since one can interpret it as establishing cause and effect, i.e., each variable is either causing the evolution (and hence this variable is an input) or producing an effect (due to the input, and hence this variable is called output). However, it can also not be denied that the input/output approach and the state-space paradigms have many important shortcomings. As an example, consider the usual procedure in modelling: a system is viewed as an interconnection of subsystems, and modelling consists of describing the individual subsystems and their interconnections laws. The result of such a modelling

This work was supported in part by the Portuguese Foundation for Science and Technology (FCT-Fundação para a Ciência e a Tecnologia), through CIDMA - Center for Research and Development in Mathematics and Applications, within project UID/MAT/04106/2013 and also by Project POCI-01-0145-FEDER-006933 - SYSTEC - Research Center for Systems and Technologies - funded by FEDER funds through COMPETE2020 - Programa Operacional Competitividade e Internacionalização (POCI) - and by national funds through FCT - Fundação para a Ciência e a Tecnologia.

978-1-5386-5346-3/18/\$31.00 (C) 2018 IEEE

\author{
José Carlos Aleixo \\ Department of Mathematics \\ University of Beira Interior \\ Covilhã, Portugal \\ jcaleixo@ubi.pt
}

procedure will be a model containing high order differential equations which involves manifest variables (the variables we try to model) and latent variables (the variables describing the subsystems and their interconnections). Consequently, in order to obtain first order or transfer function models, some manipulation has to be performed or physical insight into the structure of the system must be used. Such manipulations can become very awkward for complicated systems.

In [1], J.C. Willems proposed a paradigm for the description of systems that covers the input-output and the state-space one, and overcomes their inadequacies. This framework relies on the idea that control systems are described by equations, but their properties of interest are most naturally expressed in terms of the set of all solutions to the equations. This is formalized by the relatively new notion of system behavior yielding the so-called behavioral approach to systems theory. This approach, and in particular kernel and image representations, supplies an effective framework for modelling, where latent variables come up naturally. Examples and a thorough introduction to these ideas can be found in [2].

Recently, interesting results have been obtained in the theory of periodic behaviors, [3], [4], in particular concerning the state-space realization of periodic kernel behaviors, i.e., of behaviors described as solution sets of linear difference equations with periodically time-varying coefficients. Indeed, it was shown how to obtain periodic state space representations for periodic kernel behavioral systems using a lifting technique which allows to associate a time-invariant behavior to a periodic one.

Here we continue this line of work and address the realization problem of periodic linear systems using the behavioral framework. In particular, we concentrate on a problem that has not been previously encountered, namely, the state-space 
realization of periodic image behaviors. Such behaviors are described as the image of polynomial shift operators with periodically time-varying coefficients. They constitute an interesting class of behaviors due, among others, to their connection with convolutional codes [5]. Our approach differs from the classical one, see for instance [6] and references therein, since we do not start from a transfer function description but rather from linear difference equations with periodically time-varying coefficients.

We first observe, and illustrate by means of an example, that the periodic state-space model resulting from separately realizing each of the time-invariant image behaviors obtained by "freezing" the time-varying coefficients does not necessarily yield the same behavior. However, we show that if the "frozen" time-invariant image representations possess the same column degrees, then the former periodic realization does produce the original periodic behavior. Moreover, in the case of different columns degrees, we still provide a method to obtain a periodic state-space realization for a given periodic image behavior.

\section{PReliminaries}

\section{A. Behavioral systems}

Definition 1. A dynamical system $\Sigma$ is defined [7], [8] as a triple of sets

$$
\Sigma=(\mathbb{T}, \mathbb{W}, \mathcal{B}),
$$

where $\mathbb{T}$ is called the time axis, $\mathbb{W}$ is the signal space, and $\mathcal{B}$ is a subset of $\mathbb{W}^{\mathbb{T}}=\{f: \mathbb{T} \rightarrow \mathbb{W}\}$ called the system behavior. The elements of $\mathcal{B}$ are called (system) trajectories.

We define the backwards-shift operator by $\left(\sigma^{-1} w\right)(t)=$ $w(t-1)$, for $t \in \mathbb{Z}$.

In this paper $\mathbb{T}=\mathbb{Z}$ and $\mathbb{W}=\mathbb{R}^{q}$, for some $q \in \mathbb{N}$, i.e., we consider discrete-time systems. We focus our attention on behaviors consisting of trajectories with finite support and that, moreover, can be defined in terms of image representations as follows.

Definition 2. A system $\Sigma=\left(\mathbb{Z}, \mathbb{R}^{q}, \mathcal{B}\right)$ and the corresponding behavior $\mathcal{B}$ are said to have an image representation if $\mathcal{B}$ can be written as

$$
\mathcal{B}=\left\{w \in \mathcal{K}^{q}: \exists v \in \mathcal{K}^{\ell} \text { s.t. } w(k)=\left(M\left(\sigma^{-1}\right) v\right)(k)\right\}
$$

where, for $r \in \mathbb{N}, \mathcal{K}^{r}$ denotes the set of finite support sequences taking values in $\mathbb{R}^{r}$, and $M\left(z^{-1}\right) \in \mathbb{R}^{q \times \ell}\left[z^{-1}\right]$ is a polynomial matrix in the indeterminate $z^{-1}$. In this case $M$ is called an image representation matrix of $\mathcal{B}$, and $\mathcal{B}$ is said to be an image behavior.

A matrix $M\left(z^{-1}\right)$ is said to be column reduced if the sum of its column degrees has the minimum value among all the matrices with the same image. The degree of a column is defined as the maximum of the degrees of its entries. Note that such degrees are considered for the indeterminate $z^{-1}$. Therefore, for instance, $z^{-2}$ has degree 2.

\section{B. Periodically time-varying behaviors}

In this paper we consider behaviors $\mathcal{B}$ with $P$-periodic image representations ( $P$-periodic image behaviors), i.e.:

$$
\begin{gathered}
\mathcal{B}=\left\{w \in \mathcal{K}^{q}: \exists v \in \mathcal{K}^{\ell} \text { s.t. (1) is satisfied }\right\} \\
w(P k+t)=\left(M^{t}\left(\sigma^{-1}\right) v\right)(P k+t) ; t=0, \ldots, P-1,
\end{gathered}
$$

where each $M^{t}\left(z^{-1}\right)$ can be regarded as a $q \times \ell$ timeinvariant image representation. Such behaviors will be called $P$-periodic, and the sequence of matrices $\left(M^{0}, \ldots, M^{P-1}\right)$ will be called a $P$-periodic image representation of $\mathcal{B}$.

Inspired by the ideas developed in [4] and [3] for the case of behaviors, considering the linear map

$$
L_{p}: \mathcal{K}^{q} \rightarrow \mathcal{K}^{P q}
$$

such that

$$
\left(L_{p} w\right)(k)=\left[\begin{array}{c}
w(P k) \\
w(P k+1) \\
\vdots \\
w(P k+P-1)
\end{array}\right], P \in \mathbb{N}
$$

we associate with $\mathcal{B}$ a time-invariant behavior $\mathcal{B}^{L}$, the lifted version of $\mathcal{B}$, defined as

$$
\mathcal{B}^{L}=\left\{\widetilde{w} \in \mathcal{K}^{P q}: \widetilde{w}=L_{p} w, w \in \mathcal{B}\right\} .
$$

Note that, since

$$
\left(M^{t}\left(\sigma^{-1}\right) v\right)(P k+t)=\left(\left(\sigma^{t} M^{t}\left(\sigma^{-1}\right)\right) v\right)(P k),
$$

the equation in (1) can also be written as

$$
\left(\Omega_{P, q}(\sigma) w\right)(P k)=\left(M\left(\sigma, \sigma^{-1}\right) v\right)(P k), k \in \mathbb{Z},
$$

where for $r \in \mathbb{N}$

$$
\Omega_{P, r}(\sigma)=\left[\begin{array}{c}
I_{r} \\
\sigma I_{r} \\
\vdots \\
\sigma^{P-1} I_{r}
\end{array}\right]
$$

is a polynomial matrix operator in the shift $\sigma$ and

$$
M\left(\sigma, \sigma^{-1}\right)=\left[\begin{array}{c}
M^{0}\left(\sigma^{-1}\right) \\
\sigma M^{1}\left(\sigma^{-1}\right) \\
\vdots \\
\sigma^{P-1} M^{P-1}\left(\sigma^{-1}\right)
\end{array}\right]
$$

is a polynomial matrix operator in the shifts $\sigma$ and $\sigma^{-1}$.

Moreover, it is possible to show that the matrix $M\left(z, z^{-1}\right)$ can be decomposed as

$$
M\left(z, z^{-1}\right)=M^{L}\left(z^{-P}\right) \Omega_{P, \ell}(z)
$$

where

$M^{L}\left(z^{-1}\right)=\left[M^{L_{0}}\left(z^{-1}\right)\left|M^{L_{1}}\left(z^{-1}\right)\right| \cdots \mid M^{L_{P-1}}\left(z^{-1}\right)\right]$ 
and the blocks $M^{L_{j}}\left(\sigma^{-1}\right)$ have size $P q \times \ell, j=0, \ldots, P-1$.

Thus, the lifted behavior can be represented as

$$
\mathcal{B}^{L}=\left\{\widetilde{w}: \widetilde{w}(k)=\left(M^{L}\left(\sigma^{-1}\right) \widetilde{u}\right)(\ell), \ell \in \mathbb{N}_{0}\right\},
$$

where $\widetilde{w}=L_{P} w$ and $\widetilde{v}=L_{P} v$.

In the following example we illustrate how to construct the matrix $M^{L}\left(z^{-1}\right)$ for the 2-periodic case, given the matrices $M^{0}\left(z^{-1}\right)$ and $M^{1}\left(z^{-1}\right)$.

Example 3. Let

$$
M^{0}\left(z^{-1}\right)=\left[\begin{array}{cc}
z^{-2}-z^{-1} & 1 \\
z^{-3} & z^{-1} \\
z^{-1}+1 & z^{-2}
\end{array}\right]
$$

and

$$
M^{1}\left(z^{-1}\right)=\left[\begin{array}{cc}
1-z^{-1} & z^{-3}-z^{-1} \\
z^{-2} & z^{-1}-z^{-2} \\
z^{-4}+z^{-1} & 1
\end{array}\right] .
$$

Applying the previous procedure we have that

$$
\begin{gathered}
{\left[\begin{array}{c}
\left.M^{0}\left(z^{-1}\right)\right] \\
z M^{1}\left(z^{-1}\right)
\end{array}\right]=\left[\begin{array}{cc}
z^{-2}-z^{-1} & 1 \\
z^{-3} & z^{-1} \\
z^{-1}+1 & z^{-2} \\
z-1 & z^{-2}-1 \\
z^{-1} & 1-z^{-1} \\
z^{-3}+1 & z
\end{array}\right]} \\
=\left[\begin{array}{cc}
z^{-2} & 1 \\
0 & 0 \\
1 & z^{-2} \\
-1 & z^{-2}-1 \\
0 & 1 \\
1 & 0
\end{array}\right]+\left[\begin{array}{cc}
-z^{-2} & 0 \\
z^{-4} & z^{-2} \\
z^{-2} & 0 \\
1 & 0 \\
z^{-2} & -z^{-2} \\
z^{-4} & 1
\end{array}\right] z
\end{gathered}
$$

and so

$$
M^{L}\left(z^{-1}\right)=\left[\begin{array}{cccc}
z^{-1} & 1 & -z^{-1} & 0 \\
0 & 0 & z^{-2} & z^{-1} \\
1 & z^{-1} & z^{-1} & 0 \\
-1 & z^{-1}-1 & 1 & 0 \\
0 & 1 & z^{-1} & -z^{-1} \\
1 & 0 & z^{-2} & 1
\end{array}\right] .
$$

\section{State-space realizations}

A state-space system

$$
\left\{\begin{array}{c}
x(k+1)=A x(k)+B v(k) \\
w(k)=C x(k)+D v(k)
\end{array}, k \in \mathbb{N}_{0},\right.
$$

denoted by $(A, B, C, D)$, where $A \in \mathbb{R}^{n \times n}, B \in \mathbb{R}^{n \times \ell}, C \in$ $\mathbb{R}^{q \times n}$ and $D \in \mathbb{R}^{q \times \ell}$, is said to be a state-space realization of the time-invariant behavior $\mathcal{B}$ if $\mathcal{B}$ is the set of finite support sequences $w$ corresponding to finite support input sequences $v$ and zero initial conditions, i.e., $x(0)=0$.

This definition implicitly assumes that $(A, B, C, D)$ is a minimal realization of $\mathcal{B}$, i.e., that $A$ has the minimal possible
State-space realizations of image behaviors can be obtained as minimal state-space realizations of column reduced image representation matrices. If $M\left(z^{-1}\right) \in \mathbb{R}^{q \times \ell}\left[z^{-1}\right]$ is an image representation of $\mathcal{B},(A, B, C, D)$ is a state-space realization of $M\left(z^{-1}\right)$ if

$$
M\left(z^{-1}\right)=C(z I-A)^{-1} B+D .
$$

If $M\left(z^{-1}\right)=\sum_{i \in \mathbb{N}} M_{i} z^{-i}$, with $M_{i} \in \mathbb{R}^{q \times \ell}$, then

$$
M_{0}=D, \quad M_{i}=C A^{i-1} B, i \geq 1 \text {. }
$$

In our case, $M\left(z^{-1}\right)$ is a polynomial matrix in $z^{-1}$ and hence $M_{i}=C A^{i-1} B=0$ for $i \geq j$, for some positive integer $j$. In particular, if the realization $(A, B, C, D)$ is minimal, the matrix $A$ is nilpotent.

Note that $M\left(z^{-1}\right)$ admits many realizations. It is well-known that a state-space realization $(A, B, C, D)$ of $M\left(z^{-1}\right)$ has minimal dimension among all the realizations of $M\left(z^{-1}\right)$ if $(A, B)$ is controllable and $(A, C)$ is observable, i.e., the polynomial matrices $\left[\begin{array}{lll}z I-A & B\end{array}\right]$ and $\left[\begin{array}{c}z I-A \\ C\end{array}\right]$ have, respectively, right and left polynomial inverses (in $z$ ). The minimal dimension of a state-space realization of $M\left(z^{-1}\right)$ is called the McMillan degree [10] of $M\left(z^{-1}\right)$ and it is represented as $\mu(M)$.

The next proposition, adapted from [11], [12], provides a statespace realization for a given (not necessarily column reduced) encoder.

Proposition 4. Let $M\left(z^{-1}\right) \in \mathbb{R}^{q \times \ell}\left[z^{-1}\right]$ be a polynomial matrix with rank $\ell$ and column degrees $\nu_{1}, \ldots, \nu_{\ell}$. Consider $\bar{n}=\sum_{i=1}^{\ell} \nu_{i}$. Let $M\left(z^{-1}\right)$ have columns $m_{i}\left(z^{-1}\right)=$ $\sum_{k=0}^{\nu_{i}} m_{k, i} z^{-k}, i=1, \ldots, \ell$ where $m_{k, i} \in \mathbb{R}^{q}$. For $i=$ $1, \ldots, \ell$ define the matrices

$$
\begin{gathered}
A_{i}=\left[\begin{array}{cccc}
0 & \cdots & \cdots & 0 \\
1 & & & \vdots \\
& \ddots & & \vdots \\
& & 1 & 0
\end{array}\right] \in \mathbb{R}^{\nu_{i} \times \nu_{i}}, B_{i}=\left[\begin{array}{c}
1 \\
0 \\
\vdots \\
0
\end{array}\right] \in \mathbb{R}^{\nu_{i}}, \\
C_{i}=\left[\begin{array}{lll}
m_{1, i} & \cdots & m_{\nu_{i}, i}
\end{array}\right] \in \mathbb{R}^{q \times \nu_{i}} .
\end{gathered}
$$

Then a state-space realization of $G$ is given by the matrix quadruple $(A, B, C, D) \in \mathbb{R}^{\bar{n} \times \bar{n}} \times \mathbb{R}^{\bar{n} \times \ell} \times \mathbb{R}^{q \times \bar{n}} \times \mathbb{R}^{q \times \ell}$ where

$$
\begin{gathered}
A=\left[\begin{array}{lll}
A_{1} & & \\
& \ddots & \\
& & A_{\ell}
\end{array}\right], B=\left[\begin{array}{ccc}
B_{1} & \\
& \ddots & \\
& & B_{\ell}
\end{array}\right], \\
C=\left[\begin{array}{lll}
C_{1} & \cdots & C_{\ell}
\end{array}\right], D=\left[\begin{array}{lll}
m_{0,1} & \cdots & m_{0, \ell}
\end{array}\right]=G(0) .
\end{gathered}
$$

In the case where $\nu_{i}=0$ the ith block is missing and in $B$ a zero column occurs. 
In this realization $(A, B)$ is controllable and if $M\left(z^{-1}\right)$ is a column reduced image representation matrix, $(A, C)$ is observable. Thus, the McMillan degree of a column reduced image representation matrix is equal to the sum of its column degrees.

\section{StATE-SPACE REALiZATIONS OF PERIODiC IMAGE BEHAVIORS}

As previously mentioned, here we concentrate on the 2periodic case.

Definition 5. Let $\Sigma_{i}=\left(A_{i}, B_{i}, C_{i}, D_{i}\right), i=0,1$, be two state-space systems with the same dimension. We define a 2-periodic state-space system $\Sigma_{p}$ as

$$
\left\{\begin{array}{c}
x(k+1)=A(k) x(k)+B(k) u(k) \\
w(k)=C(k) x(k)+D(k) v(k)
\end{array}, k \in \mathbb{N}_{0}\right.
$$

where $A(\cdot), B(\cdot), C(\cdot), D(\cdot)$ are periodic functions with period 2 , such that, for each $j \in \mathbb{N}_{0}$,

$$
(A(2 j), B(2 j), C(2 j), D(2 j))=\left(A_{0}, B_{0}, C_{0}, D_{0}\right)
$$

and

$$
(A(2 j+1), B(2 j+1), C(2 j+1), D(2 j+1))=\left(A_{1}, B_{1}, C_{1}, D_{1}\right)
$$

The dimension of $\Sigma_{p}$ is defined as the dimension of the state vector $x$. In this case we say that $\Sigma_{p}$ is obtained from $\Sigma_{0}$ and $\Sigma_{1}$.

Moreover, $\Sigma_{p}$ is a realization of a 2-periodic image representation $\left(M^{0}, M^{1}\right)$ and of the associated 2-periodic image behavior, if the output of $\Sigma_{p}$ that corresponds to an input $v$ equals the trajectory $w$ corresponding to $v$ according to $(1)$.

Let $\Sigma_{0}$ and $\Sigma_{1}$ be two state-space realizations (of the same dimension) of two time-invariant image representations $M^{0}\left(z^{-1}\right)$ and $M^{1}\left(z^{-1}\right)$. In the following example we show that the 2 -periodic system $\Sigma_{p}$ obtained from $\Sigma_{0}$ and $\Sigma_{1}$ is not, in general, a state-space realization of the associated 2-periodic image behavior (see also [13]).

Example 6. Consider the two time-invariant image behaviors with image representations

$$
\begin{aligned}
M^{0}\left(z^{-1}\right) & =M_{0}^{0}+M_{1}^{0} z^{-1}+M_{2}^{0} z^{-2} \\
& =\left[\begin{array}{ccc}
1+z^{-2} & 1 & 0 \\
z^{-2} & 1+z^{-1} & 1 \\
1+z^{-1} & 1 & 1 \\
1 & 1 & 1+z^{-1}
\end{array}\right]
\end{aligned}
$$

and

$$
\begin{aligned}
M^{1}\left(z^{-1}\right) & =M_{0}^{1}+M_{1}^{1} z^{-1}+M_{2}^{1} z^{-2} \\
& =\left[\begin{array}{ccc}
1+z^{-1} & 1 & 0 \\
1+z^{-2} & 1+z^{-1} & 1 \\
1 & 1+z^{-2} & 1 \\
0 & 1 & 1
\end{array}\right] .
\end{aligned}
$$

Realizing $M^{0}\left(z^{-1}\right)$ as in Proposition 4 we obtain the statespace realization $\Sigma_{0}=(A(0), B(0), C(0), D(0))$ with

$$
\begin{array}{ccc}
A(0) & =\left[\begin{array}{llll}
0 & 0 & 0 & 0 \\
1 & 0 & 0 & 0 \\
0 & 0 & 0 & 0 \\
0 & 0 & 0 & 0
\end{array}\right] & B(0)=\left[\begin{array}{lll}
1 & 0 & 0 \\
0 & 0 & 0 \\
0 & 1 & 0 \\
0 & 0 & 1
\end{array}\right] \\
C(0)=\left[\begin{array}{llll}
0 & 1 & 0 & 0 \\
0 & 1 & 1 & 0 \\
1 & 0 & 0 & 0 \\
0 & 0 & 0 & 1
\end{array}\right] & D(0)=\left[\begin{array}{lll}
1 & 1 & 0 \\
0 & 1 & 1 \\
1 & 1 & 1 \\
1 & 1 & 1
\end{array}\right] .
\end{array}
$$

Proceeding the same way, we obtain a state-space realization of $M^{1}\left(z^{-1}\right), \Sigma_{1}=(A(1), B(1), C(1), D(1))$, with

$$
\begin{array}{cc}
A(1)=\left[\begin{array}{llll}
0 & 0 & 0 & 0 \\
1 & 0 & 0 & 0 \\
0 & 0 & 0 & 0 \\
0 & 0 & 1 & 0
\end{array}\right] & B(1)=\left[\begin{array}{lll}
1 & 0 & 0 \\
0 & 0 & 0 \\
0 & 1 & 0 \\
0 & 0 & 0
\end{array}\right] \\
C(1)=\left[\begin{array}{llll}
1 & 0 & 0 & 0 \\
0 & 1 & 1 & 0 \\
0 & 0 & 0 & 1 \\
0 & 0 & 0 & 0
\end{array}\right] & D(1)=\left[\begin{array}{lll}
1 & 1 & 0 \\
1 & 1 & 1 \\
1 & 1 & 1 \\
0 & 1 & 1
\end{array}\right] .
\end{array}
$$

Let us consider $v$ such that $v(0)=v_{0}, v(1)=v_{1}, v(k)=0$, $k \geq 2$, with $v_{0}=\left[\begin{array}{l}0 \\ 0 \\ 1\end{array}\right]$ and $v_{1}=\left[\begin{array}{l}0 \\ 0 \\ 0\end{array}\right]$. From (1) it follows that

$w_{1}=M_{0}^{1} v_{1}+M_{1}^{1} v_{0}=\left[\begin{array}{ccc}1 & 1 & 0 \\ 1 & 1 & 1 \\ 1 & 1 & 1 \\ 0 & 1 & 1\end{array}\right] v_{1}+\left[\begin{array}{lll}1 & 0 & 0 \\ 0 & 1 & 0 \\ 0 & 0 & 0 \\ 0 & 0 & 0\end{array}\right] v_{0}=\left[\begin{array}{l}0 \\ 0 \\ 0 \\ 0\end{array}\right]$

while from (3)

$$
\begin{gathered}
w_{1}=D(1) v_{1}+C(1) B(0) v_{0} \\
=\left[\begin{array}{lll}
1 & 1 & 0 \\
1 & 1 & 1 \\
1 & 1 & 1 \\
0 & 1 & 1
\end{array}\right] v_{1}+\left[\begin{array}{llll}
1 & 0 & 0 & 0 \\
0 & 1 & 1 & 0 \\
0 & 0 & 0 & 1 \\
0 & 0 & 0 & 0
\end{array}\right]\left[\begin{array}{lll}
1 & 0 & 0 \\
0 & 0 & 0 \\
0 & 1 & 0 \\
0 & 0 & 1
\end{array}\right] v_{0}=\left[\begin{array}{l}
0 \\
0 \\
1 \\
0
\end{array}\right],
\end{gathered}
$$

i.e., the output $w$ of the periodic state-space system $\Sigma_{p}$ obtained from $\Sigma_{0}$ and $\Sigma_{1}$ corresponding to $v$ is different from the trajectory $w$ corresponding to $v$ according to (1).

A possible method to overcome this problem is to: (i) obtain the lifted time-invariant image behavior $\mathcal{B}^{L}$ associated with the 2-periodic image behavior $\mathcal{B}$, as shown in Section II-B; (ii) construct a time-invariant state-space realization $\Sigma^{L}=\left(A^{L}, B^{L}, C^{L}, D^{L}\right)$ for $\mathcal{B}^{L}$; (iii) try to obtain a 2periodic state-space realization $\Sigma_{p}=(A(\cdot), B(\cdot), C(\cdot), D(\cdot))$ for $\mathcal{B}$ using a similar method as proposed in [3].

This method consists in the suitable factorization of the matrices $A^{L}, B^{L}, C^{L}$ and $D^{L}$ so as to obtain $A(0), A(1)$, 
$B(0), B(1), C(0), C(1), D(0)$ and $D(1)$. In fact, it can be shown that if

$$
\begin{array}{rlrl}
A^{L} & =A(1) A(0) & B^{L} & =\left[\begin{array}{cc}
A(1) B(0) & B(1)
\end{array}\right] \\
C^{L} & =\left[\begin{array}{c}
C(0) \\
C(1) A(0)
\end{array}\right] & D^{L}=\left[\begin{array}{cc}
D(0) & 0 \\
C(1) B(0) & D(1)
\end{array}\right]
\end{array}
$$

then $\Sigma_{p}=(A(\cdot), B(\cdot), C(\cdot), D(\cdot))$ with $A(i)=A_{i}$, $B(i)=B_{i}, C(i)=C_{i}, D(i)=D_{i}, i=0,1$ is a 2-periodic state-space realization of $\mathcal{B}$ [3]. Clearly, this factorization is not always easy to perform.

As an alternative, one can also investigate under which conditions the procedure proposed in Example 6 does yield a 2 -periodic state-space realization of a 2-periodic behavior $\mathcal{B}$. In the next theorem we provide a sufficient condition for this to hold.

Theorem 7. Consider two image representations $M^{0}\left(z^{-1}\right) \in$ $\mathbb{R}^{q \times \ell}\left[z^{-1}\right]$ and $M^{1}\left(z^{-1}\right) \in \mathbb{R}^{q \times \ell}\left[z^{-1}\right]$ with the same column degrees and let $\Sigma_{i}$ be the realizations of $M^{i}\left(z^{-1}\right), i=$ 0,1 obtained by Proposition 4. Then, the periodic statespace system $\Sigma_{p}$ obtained from $\Sigma_{0}$ and $\Sigma_{1}$ is a realization of the periodic image representation $\left(M^{0}, M^{1}\right)$ (and of the corresponding 2-periodic image behavior).

Proof: Let us denote by $\Sigma_{0}=\left(A_{0}, B_{0}, C_{0}, D_{0}\right)$ and by $\Sigma_{1}=\left(A_{1}, B_{1}, C_{1}, D_{1}\right)$ the state-space realizations of $M^{0}\left(z^{-1}\right)$ and $M^{1}\left(z^{-1}\right)$ as in Proposition 4. Since the structure of the matrices $A_{0}, A_{1}, B_{0}$ and $B_{1}$ depends only of the column degrees of $M^{0}\left(z^{-1}\right)$ and $M^{1}\left(z^{-1}\right)$, we have that $A_{0}=A_{1}$ and $B_{0}=B_{1}$. Then the 2-periodic state-space system obtained from $\Sigma_{0}$ and $\Sigma_{1}$ has updating equations

$$
\left\{\begin{array}{cl}
x(k+1) & =A x(k)+B v(k) \\
w(k) & =C_{k-2\left\lfloor\frac{k}{2}\right\rfloor} x(k)+D_{k-2\left\lfloor\frac{k}{2}\right\rfloor} v(k)
\end{array}, k \in \mathbb{N}_{0},\right.
$$

where $A=A_{0}=A_{1}$ and $B=B_{0}=B_{1}$. Therefore, it follows from (2) that for any $v \in \mathcal{K}^{\ell}$,

$$
\begin{aligned}
w(k) & =D_{0} v(k)+\sum_{i=1}^{k} C_{0} A^{i-1} B v(k-i) \\
& =\left(M^{0}\left(\sigma^{-1}\right) v\right)(k), \text { for } k=2 t
\end{aligned}
$$

and

$$
\begin{aligned}
w(k) & =D_{1} v(k)+\sum_{i=1}^{k} C_{1} A^{i-1} B v(k-i) \\
& =\left(M^{1}\left(\sigma^{-1}\right) v\right)(k), \text { for } k=2 t+1
\end{aligned}
$$

for $t \in \mathbb{N}_{0}$, i.e., $\Sigma_{p}$ is a 2-periodic realization of the periodic image representation $\left(M^{0}, M^{1}\right)$.

In case $M^{0}\left(z^{-1}\right)$ and $M^{1}\left(z^{-1}\right)$ have different column degrees the following procedure can be applied in order to obtain a 2-periodic state-space realization of the periodic the periodic image representation $\left(M^{0}, M^{1}\right)$ from state-space realizations of $M^{0}\left(z^{-1}\right)$ and $M^{1}\left(z^{-1}\right)$ :
1) Let $\nu_{i}$ be the maximum degree of the $i$-th columns of $M^{0}\left(z^{-1}\right)$ and $M^{1}\left(z^{-1}\right), i=1, \ldots, \ell$;

2) Realize $M^{0}\left(z^{-1}\right)$ and $M^{1}\left(z^{-1}\right)$ as in Proposition 4 considering the columns of $M^{j}\left(z^{-1}\right)$ as $m_{i}^{j}\left(z^{-1}\right)=$ $\sum_{k=0}^{\nu_{i}} m_{k, i}^{j} z^{-k}, i=1, \ldots, \ell$, where some of the coefficients of higher degree may be zero.

Using this and the same line of arguments as in the proof of Theorem 7 the following theorem is immediate.

Theorem 8. Let $M^{0}\left(z^{-1}\right), M^{1}\left(z^{-1}\right) \in \mathbb{R}^{q \times \ell}\left[z^{-1}\right]$ be two image representations with state-space realizations $\Sigma_{0}$ and $\Sigma_{1}$, respectively, obtained from the procedure above. Then the 2periodic system obtained from $\Sigma_{0}$ and $\Sigma_{1}$ is a state-space realization of the periodic image representation $\left(M^{0}, M^{1}\right)$.

Example 9. Consider again the image representations of Example 6

$$
\begin{aligned}
M^{0}\left(z^{-1}\right) & =M_{0}^{0}+M_{1}^{0} z^{-1}+M_{2}^{0} z^{-2} \\
& =\left[\begin{array}{ccc}
1+z^{-2} & 1 & 0 \\
z^{-2} & 1+z^{-1} & 1 \\
1+z^{-1} & 1 & 1 \\
1 & 1 & 1+z^{-1}
\end{array}\right]
\end{aligned}
$$

and

$$
\begin{aligned}
M^{1}\left(z^{-1}\right) & =M_{0}^{1}+M_{1}^{1} z^{-1}+M_{2}^{1} z^{-2} \\
& =\left[\begin{array}{ccc}
1+z^{-1} & 1 & 0 \\
1+z^{-2} & 1+z^{-1} & 1 \\
1 & 1+z^{-2} & 1 \\
0 & 1 & 1
\end{array}\right] .
\end{aligned}
$$

Let $\nu_{1}=2, \quad \nu_{2}=2, \quad \nu_{3}=1$ be the maximum degrees of the first, second and third columns, respectively, of $M^{0}\left(z^{-1}\right)$ and $M^{1}\left(z^{-1}\right)$. The state-space realizations $\Sigma_{0}=(A, B, C(0), D(0))$ and $\Sigma_{1}=(A, B, C(1), D(1))$ of $M^{0}\left(z^{-1}\right)$ and $M^{1}\left(z^{-1}\right)$, respectively, obtained from the procedure above, are such that

$$
A=\left[\begin{array}{lllll}
0 & 0 & 0 & 0 & 0 \\
1 & 0 & 0 & 0 & 0 \\
0 & 0 & 0 & 0 & 0 \\
0 & 0 & 1 & 0 & 0 \\
0 & 0 & 0 & 0 & 0
\end{array}\right] \quad B=\left[\begin{array}{lll}
1 & 0 & 0 \\
0 & 0 & 0 \\
0 & 1 & 0 \\
0 & 0 & 0 \\
0 & 0 & 1
\end{array}\right]
$$

$$
\begin{array}{ccc}
C(0) & =\left[\begin{array}{lllll}
0 & 1 & 0 & 0 & 0 \\
0 & 1 & 1 & 0 & 0 \\
1 & 0 & 0 & 0 & 0 \\
0 & 0 & 0 & 0 & 1
\end{array}\right] & D(0)=\left[\begin{array}{lll}
1 & 1 & 0 \\
0 & 1 & 1 \\
1 & 1 & 1 \\
1 & 1 & 1
\end{array}\right] \\
C(1)=\left[\begin{array}{lllll}
1 & 0 & 0 & 0 & 0 \\
0 & 1 & 1 & 0 & 0 \\
0 & 0 & 0 & 1 & 0 \\
0 & 0 & 0 & 0 & 0
\end{array}\right] \quad D(1)=\left[\begin{array}{lll}
1 & 1 & 0 \\
1 & 1 & 1 \\
1 & 1 & 1 \\
0 & 1 & 1
\end{array}\right] .
\end{array}
$$

The 2-periodic system obtained from $\Sigma_{0}$ and $\Sigma_{1}$ is a state-space realization of the periodic image representation $\left(M^{0}, M^{1}\right)$. 


\section{CONCLUSIONS}

In this paper we have studied the realization of periodic image behaviors by periodic state-space models for the particular case of period 2. We showed that this issue is not as straightforward as it seems. In fact, separate state-space realizations of each of the associated time-invariant image behaviors does not necessarily yield a periodic state-space realization of the corresponding periodic image behavior. However, we presented a direct method to obtain a periodic state-space realization provided certain conditions are satisfied. The analysis of minimality issues is an interesting question and is currently being studied.

\section{REFERENCES}

[1] J. Willems, "Paradigms and puzzles in the theory of dynamical systems," IEEE Trans. Automat. Control, vol. 36, no. 3, pp. 259-294, 1991.

[2] J. Polderman and J. Willems, Introduction to mathematical systems theory: a behavioral approach, ser. Texts in Applied Mathematics. New York NY, USA: Springer, 1997, vol. 26.

[3] J. C. Aleixo, P. Rocha, and J. C. Willems, "State space representation of siso periodic behaviors," in 2011 50th IEEE Conference on Decision and Control and European Control Conference, Dec 2011, pp. 1545-1550.

[4] M. Kuijper and J. C. Willems, "A behavioral framework for periodically time-varying systems," in Proceedings of the 36th IEEE Conference on Decision and Control, vol. 3, Dec 1997, pp. 2013-2016 vol.3.

[5] J. Rosenthal, "Connections between linear systems and convolutional codes," in Codes, Systems and Graphical Models, ser. IMA Vol. 123, B. Marcus and J. Rosenthal, Eds. Springer-Verlag, 2001, pp. 39-66.

[6] E. Snchez, V. Hernndez, and R. Bru, "Minimal realizations for discrete-time linear periodic systems," Linear Algebra and its Applications, vol. 162-164, pp. 685 - 708, 1992. [Online]. Available: http://www.sciencedirect.com/science/article/pii/002437959290402V

[7] J. Willems, "Models for dynamics," in Dynamics reported, Vol. 2, ser. Dynam. Report. Ser. Dynam. Systems Appl. Chichester: Wiley, 1989, vol. 2, pp. 171-269.

[8] J. Polderman and J. Willems, Introduction to Mathematical Systems Theory, A behavioral approach, ser. Texts in Applied Mathematics. New York: Springer-Verlag, 1998, vol. 26.

[9] J. Rosenthal and E. V. York, "BCH convolutional codes," IEEE Trans. Automat. Control, vol. 45, no. 6, pp. 1833-1844, 1999.

[10] T. Kailath, Linear systems, ser. Prentice Hall information and system sciences series. Englewood Cliffs: Prentice-Hall, 1980.

[11] E. Fornasini and R. Pinto, "Matrix fraction descriptions in convolutional coding," Linear Algebra and its Applications, vol. 392, no. Supplement C, pp. 119 - 158, 2004. [Online]. Available: http://www.sciencedirect.com/science/article/pii/S0024379504002836

[12] H. Gluesing-Luerssen and G. Schneider, "State space realizations and monomial equivalence for convolutional codes," Linear Algebra and its Applications, vol. 425, no. 2, pp. 518 - 533, 2007, special Issue in honor of Paul Fuhrmann. [Online]. Available: http://www.sciencedirect.com/science/article/pii/S002437950700122X

[13] J. C. Aleixo and P. Rocha, "Roesser model representation of $2 \mathrm{~d}$ periodic behaviors: the (2,2)-periodic siso case," in 2017 10th International Workshop on Multidimensional (nD) Systems (nDS), Sept 2017, pp. 1-6. 